\title{
RiboCas: A Universal CRISPR-Based Editing Tool for Clostridium
}

\author{
Inés C. Cañadas, ${ }^{\dagger}$ Daphne Groothuis, ${ }^{\dagger}$ Maria Zygouropoulou, ${ }^{\dagger}$ Raquel Rodrigues, ${ }^{\dagger}$ \\ and Nigel P. Minton*, $\uparrow, \ddagger$ (1)
}

${ }^{\dagger}$ Clostridia Research Group, BBSRC/EPSRC Synthetic Biology Research Centre (SBRC), School of Life Sciences, Centre for Biomolecular Sciences, The University of Nottingham, Nottingham NG7 2RD, U.K.

${ }^{\ddagger}$ NIHR Nottingham Biomedical Research Centre, Nottingham University Hospitals NHS Trust and the University of Nottingham, Nottingham NG7 2RD, U.K.

\section{Supporting Information}

ABSTRACT: Members of the genus Clostridium represent a diverse assemblage of species exhibiting both medical and industrial importance. Deriving both a greater understanding of their biology, while at the same time enhancing their exploitable properties, requires effective genome editing tools. Here, we demonstrate the first implementation in the genus of theophylline-dependent, synthetic riboswitches exhibiting a full set of dynamic ranges, also suitable for applications where tight control of gene expression is required. Their utility was highlighted by generating a novel riboswitch-based editing tool-RiboCas - that overcomes the main obstacles associ-

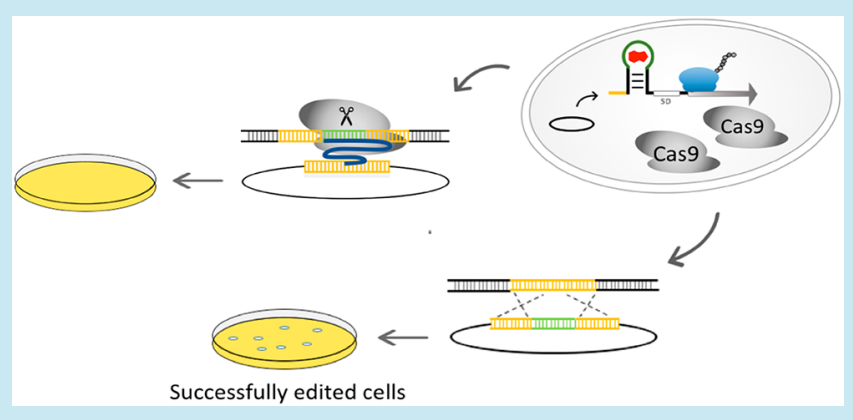
ated with CRISPR/Cas9 systems, including low transformation efficiencies and excessive Cas9 toxicity. The universal nature of the tool was established by obtaining chromosomal modifications in C. pasteurianum, C. difficile, and C. sporogenes, as well as by carrying out the first reported example of CRISPR-targeted gene disruption in C. botulinum. The high efficiency (100\% mutant generation) and ease of application of RiboCas make it suitable for use in a diverse range of microorganisms.

KEYWORDS: Clostridium, riboswitch, inducible expression, CRISPR, synthetic biology

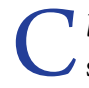
lostridium is a large genus of Gram-positive, anaerobic, spore-forming bacteria that includes species relevant to human and animal diseases as well as representatives with application in biotechnological processes. ${ }^{1}$ Those members of the genus that cause disease include the notorious pathogens Clostridium botulinum, ${ }^{2}$ Clostridium perfringens, ${ }^{3}$ and Clostridium difficile. ${ }^{4}$ The majority of the genus are, however, entirely benign and form the basis of commercial processes concerned with the production of platform chemicals and biofuels (e.g., Clostridium pasteurianum, ${ }^{5,6}$ Clostridium acetobutylicum ${ }^{7}$ ); cellulosic and hemicellulosic biomass degradation (e.g., Clostridium thermocellum ${ }^{8}$ ); carbon fixation (e.g., Clostridium ljungdahlii ${ }^{9}$ and Clostridium autoethanogenum $\left.{ }^{10}\right)$; and anticancer therapeutics (e.g., Clostridium sporogenes ${ }^{1,12}$ ). Regardless of their extraordinary diversity, deriving both a greater understanding of their biology, while at the same time enhancing their exploitable properties, requires ever more sophisticated approaches. This challenge is met by the emerging field of synthetic biology, which can provide common tools for engineering new functionalities in pathogens and industrial strains alike.

Adding to a growing list of available synthetic biology tools, are those based on the Streptococcus pyogenes CRISPR/Cas9 system (clustered regularly interspaced short palindromic repeats/CRISPR-associated proteins) which can form the basis of rapid and reliable methods for genome editing. In prokaryotes, genome editing is reliant on recombination-based replacement of a parental sequence with the desired mutant allele and subsequent elimination of the progenitor cell population through RNA-guided, Cas9 cleavage of the parental allele. It follows that in the ideal system, a tightly regulated promoter should be used to initially prevent production of Cas9 until the allelic exchange necessary to generate the desired mutant cell population has taken place. Restricting the time during which Cas9 is present also reduces the opportunity for potential off target effects and the likelihood of mutations that inactivate Cas9 or the sgRNA. Those systems described in Clostridium species to date have largely used constitutive promoters to control Cas9 production or promoters that are poorly repressed. ${ }^{13-18}$ In general, their effectiveness has been hindered by low transformation and/or editing efficiencies. The use of a Cas9 nickase (nCas9), a Cas9 mutant that introduces a breakage on only one strand of the chromosome, ${ }^{19}$ has been shown to enable the isolation of Clostridium mutants. $^{20,21}$ However, because nCas9 has a less powerful selection capacity than Cas9, the isolation of mutants becomes more time-consuming, requiring several passages onto fresh medium. $^{20}$

Received: February 20, 2019

Published: June 7, 2019 
a

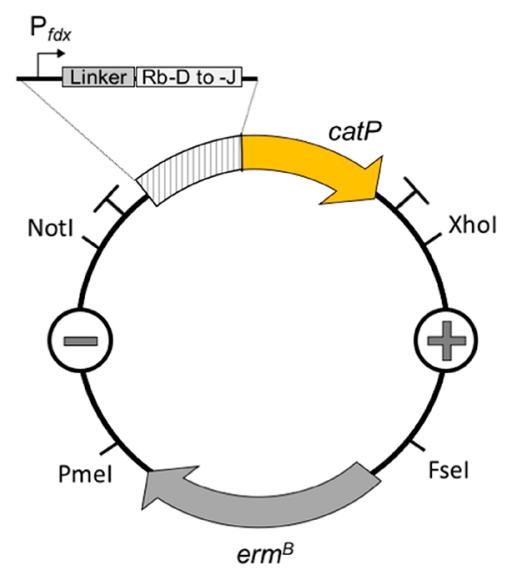

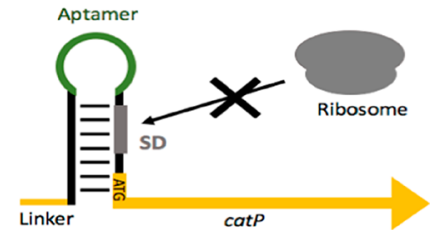

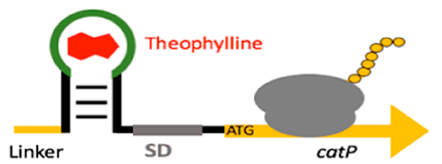

Figure 1. E. coli/Clostridium shuttle plasmid pMTL-IC101 and theophylline-riboswitch gene-control function. (a) E. coli/Clostridium shuttle plasmid pMTL-IC101 containing the catP reporter gene under the control of $\mathrm{P}_{f d x}$. Riboswitches-D to -J (Rb-D to $\left.-\mathrm{J}\right)$ were placed downstream of the TSS. (-) is the Gram-negative ColE1 RNA II origin of replication that allows replication of the shuttle plasmid in E. coli. (+) is the Grampositive replicon derived from the Clostridium botulinum plasmid pBP1. (b) Schematic representation of a functional model of the theophylline responsive riboswitch. In the absence of theophylline, the riboswitch forms a stem-loop structure that sequesters the ribosome binding site (RBS) in the mRNA transcript. When theophylline binds to the aptamer, the riboswitch conformation changes resulting in the release of the RBS and initiation of translation of the gene of interest (catP).

a

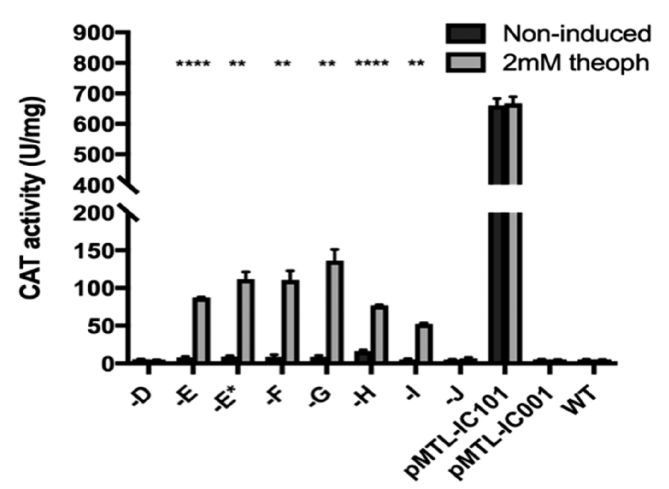

b

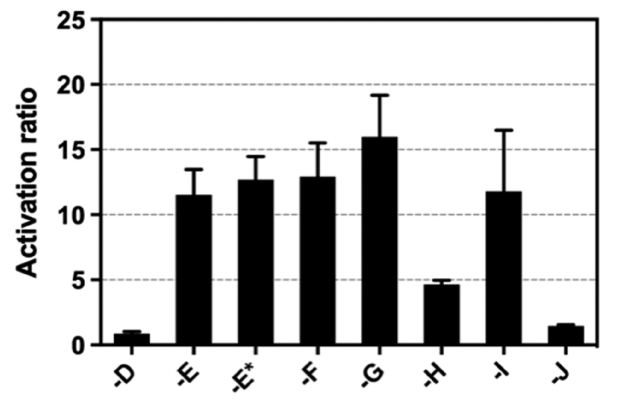

Figure 2. Theophylline-riboswitch performance in Clostridium. (a) CAT activity and its ligand-dependent induction in each pMTL-IC111 reporter plasmid. The reporter plasmids pMTL-IC101 $\left(\mathrm{P}_{f d x}-c a t P\right)$, pMTL-IC001 (promoterless catP), as well as the WT strain were used as controls. Error bars represent standard deviations of three biological replicates. Asterisks indicate statistically significant induction values for $* p \leq 0.0332$, $* * p \leq$ $0.0021, * * * p \leq 0.0002, * * * * p \leq 0.0001$ (paired two-tailed Student's $t$-test). (b) Activation ratio of riboswitches $-\mathrm{D}$ to $-\mathrm{J}$. The activation ratio in each riboswitch-based reporter plasmid was calculated by dividing the value of CAT activity measured in the presence of the inducer by the value of that in the absence of inducer.

To address these limitations, we sought to exploit the versatility of bacterial riboswitches to engineer a ligandresponsive system with the adequate ON/OFF states for CRISPR-based applications. These natural genetic control elements, located primarily within the $5^{\prime}$ untranslated region of mRNA ( 5 ' UTR), regulate gene expression in a liganddependent manner and in the absence of protein factors. ${ }^{22}$ Riboswitches can also be engineered to respond to specific ligands and are therefore of great benefit for synthetic biology applications. In particular, the well characterized synthetic theophylline responsive riboswitch, ${ }^{23}$ has been shown to work successfully in diverse bacterial species, ${ }^{24-29}$ suggesting its potential transferability to other prokaryotes.

The strong repression and dynamic range of some of the theophylline responsive riboswitches obtained in this study allowed the development of a novel and highly efficient CRISPR system, named RiboCas, which regulates the expression of Cas9 at the level of translation. We further demonstrate the universality of RiboCas by obtaining chromosomal modifications in the four different clostridial species C. sporogenes, C. pasteurianum, C. difficile, and C. botulinum. We suggest that the high efficiency and easy application of RiboCas make it a versatile genome editing tool, applicable to diverse microorganisms and synthetic biology endeavors.

\section{RESULTS}

Riboswitches Demonstrate Theophylline Dependency in Clostridium. As previous work had shown that many diverse theophylline-dependent riboswitches were functional in both Gram-positive and Gram-negative bacteria, ${ }^{28}$ we first sought to evaluate the performance of this inducible translational regulatory system in C. sporogenes NCIMB 10696. We selected three riboswitch sequences, riboswitch-D, -E, and -E*, which were expected to exhibit higher translation efficiencies in the "on" state, out of the six theophyllineresponsive riboswitches developed by Gallivan and coworkers. $^{23,28}$ In previous publications, riboswitch-E* was 
a

$\mathrm{P}_{f d x}-\mathrm{Rb}$

-10
TATAAAAATTACTTAAAAATTAATAAAAACATGGTAAATATA
+1
AATCLinker Riboswitch ATG gene of interest

$\mathrm{P}_{f d \times 4}-\mathrm{Rb} \quad-35$

TATAAAAAAAACTIAACTTCATG ITAAAAACTTGTTAAAATATA

$\stackrel{+1}{A A T C}$ Linker Riboswitch ATG gene of interest $\downarrow$

$\mathrm{P}_{f d x}{ }^{*}-\mathrm{Rb}$

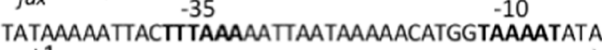

$\stackrel{+1}{+1}$ ATCGTATAAAGTIGTGTAATIII Linker Riboswitch native 5'UTR b

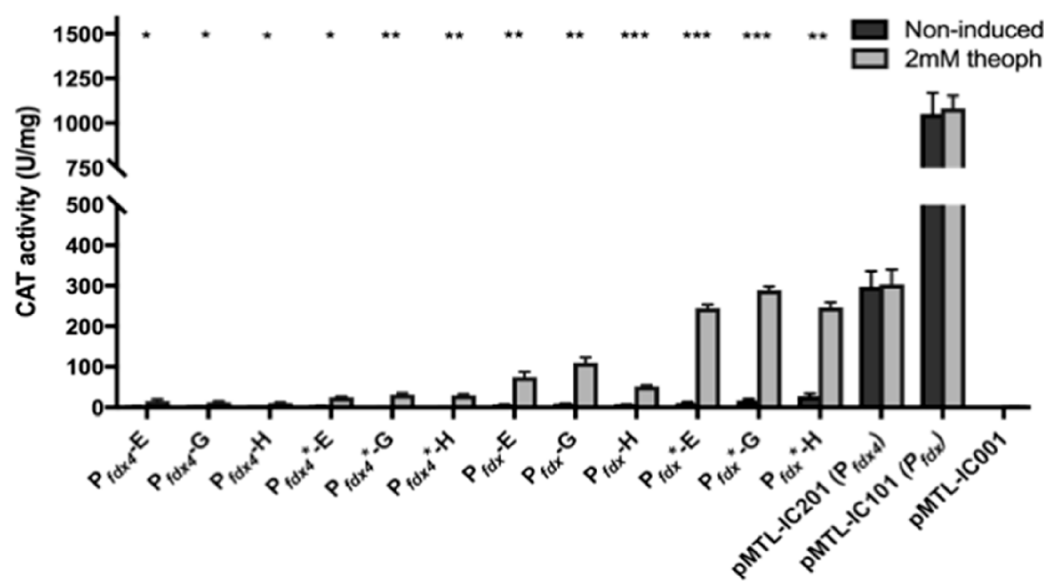

Figure 3. Tuned response of theophylline-riboswitches. (a) The sequences of the constructed theophylline-responsive switches. The predicted -35 and -10 sequences are in bold. The experimentally determined TSS via $5^{\prime}$ RACE (Figure S2) are indicated with +1 . The $5^{\prime}$ UTR sequences downstream of the TSS are underlined. Core elements replaced to create the synthetic hybrid promoter $\mathrm{P}_{f d x 4}$ are in red. (b) Library of theophyllinedependent riboswitches tested in C. sporogenes in the presence and absence of the inducer. The reporter plasmids pMTL-IC201 ( $\mathrm{P}_{f d x 4}$-catP), pMTL-IC101 $\left(\mathrm{P}_{f d x}-c a t P\right)$, and pMTL-IC001 (promoterless catP) were used as controls. Error bars represent standard deviations of three biological replicates. Asterisks indicate statistically significant induction values (paired two-tailed Student's t-test).

shown to be the best theophylline-dependent riboregulator in Gram-positive bacteria, thanks to its high dynamic range and its very low basal expression. ${ }^{25-27,29}$ Accordingly, four new riboswitches (named $-\mathrm{F}$ to $-\mathrm{I}$ ) were constructed by rationally modifying the space between the Shine-Dalgarno sequence (SD) and the translational start site of the original riboswitch$\mathrm{E}^{*}$ (Table S1). A reporter plasmid, pMTL-IC101, derived from the vector pMTL82251, ${ }^{30}$ was used as a chassis for all of the subsequently created plasmids (Figure 1a). This backbone contains the native promoter of the $C$. sporogenes ferredoxin gene $\left(\mathrm{P}_{f d x}\right.$, associated with the protein coding gene Clspo c0087) upstream of catP, which encodes the reporter enzyme Chloramphenicol Acetyl Transferase (CAT, EC: 2.3.1.28) and serves as a reference to compare CAT expression measurements. As a default, the riboswitches, preceded by a linker sequence, were genetically fused to the core region $(-35$ and -10) of the strong $\mathrm{P}_{f d x}$, just downstream of the transcription start site (TSS) and excluding the native $5^{\prime}$ UTR sequence, as previously described ${ }^{25}$ (Figure S1). Plasmid constructs, designated PMTL-IC111-D to -J, were conjugated into C. sporogenes and cultures of the resultant transconjugants cells exposed to $2 \mathrm{mM}$ theophylline inducer when they had reached early exponential growth phase $\left(\mathrm{OD}_{600} \approx 0.5\right)$. CAT activity was determined in cell lysates derived from stationary phase cultures cultivated in the presence or absence of the inducer. As a control, a promoter-less backbone, pMTL-IC001, was assembled to detect background reporter gene expression. A detailed list of plasmids used in this study is provided in Table S5.

The synthetic theophylline responsive riboswitch is composed of an aptamer and a synthetic SD (Figure 1b). Theoretically, transcription of the riboswitch under the control of $\mathrm{P}_{f d x}$ occurs in a constitutive manner during cell growth. However, the synthetic SD sequence located downstream of the aptamer is sequestered via pairing with the stem of the riboswitch, resulting in translational block. The binding of theophylline releases the SD by altering the downstream base pairing. As a consequence, gene translation occurs when the ribosome binds to the SD. ${ }^{23}$ As shown in Figure 2a, strains harboring riboswitches $-\mathrm{E},-\mathrm{E}^{*},-\mathrm{F},-\mathrm{G},-\mathrm{H}$, and -I exhibited statistically significant induction in $C$. sporogenes after addition of $2 \mathrm{mM}$ theophylline to the culture. In particular, riboswitch$\mathrm{G}$ outperformed previous theophylline-dependent riboswitches, demonstrating higher levels of CAT activity, low leakage expression and the strongest activation ratio (Figure $2 \mathrm{~b})$. In all cases, the incorporation of any riboswitch in the $5^{\prime}$ UTR led to a strong reduction in CAT activity; this agrees with previously published studies, indicating that secondary structures near the RBS play a major role in the translation of the downstream mRNA. ${ }^{23,25,27}$ CAT activity of cells harboring the control pMTL-IC001 was similar to that from cultures lacking the reporter backbone (wildtype, WT), indicating no detectable transcriptional read-through.

Riboswitches Can Be Adjusted to the Desired Regulatory Window. Due to the importance of having inducible systems where gene expression can be controlled within a defined regulatory window, we sought to compare the expression pattern of riboswitches $-\mathrm{E},-\mathrm{G}$, and $-\mathrm{H}$ when placed downstream of the core region of a synthetically weakened $\mathrm{P}_{f d x}$, named $\mathrm{P}_{f d x 4}$. $\mathrm{P}_{f d x 4}$ was generated by replacing the core elements -10 and -35 of $\mathrm{P}_{f d x}$ with the same regulatory elements of the constitutive promoter from the C. acetobutylicum ATCC 824 $p t b$ gene $\left(\mathrm{P}_{p t b}\right.$, associated with the protein coding gene $\mathrm{Ca}$ 3076) (Figure 3a). Also, to further examine the regulatory response of the theophylline responsive riboregulators in Clostridia, riboswitches $-\mathrm{E},-\mathrm{G}$ and $-\mathrm{H}$ were fused to the promoters $\mathrm{P}_{f d x}$ and $\mathrm{P}_{f d x 4}$ retaining the bases downstream the TSS but excluding their native SD sequences, as previously described $^{24}$ (Figure $\mathrm{S} 1$ ). These sequences, named $\mathrm{P}_{f d x} *$ and $\mathrm{P}_{f d x 4} *$, maintain the full upstream region, including the core region $(-35$ and -10$)$, the TSS and the space between the TSS and the native SD. Constructs were designed to express the reporter gene cat $P$, conjugated into $C$. sporogenes and growing cultures of the transconjugants obtained exposed to 2 $\mathrm{mM}$ of theophylline inducer. As shown in Figure $3 \mathrm{~b}$, the combination of different riboswitches with various promoters and two different 5' UTRs allows the expansion of the regulatory range, providing a library of theophylline-inducible 
switches suitable for applications where protein yield is crucial as well as for the expression of detrimental or toxic proteins. The combination of any riboswitch with the weak promoter $\mathrm{P}_{f d x 4}$ led to the lowest expression levels. This was also the case when the native 5' UTR sequence was retained, establishing a direct correlation between detected CAT activity and promoter strength $\left(\mathrm{P}_{f d x}>\mathrm{P}_{f d x 4}\right)$. However, basal expression appears to be compromised when high expression levels are achieved, resulting in increased leakiness in those constructs that exhibited higher expression levels (i.e., with $\mathrm{P}_{f d x}{ }^{*}-\mathrm{G}$ ). Expression of cat $P$ was always higher when the native sequence downstream of the TSS was maintained.

Gene Expression and Transcript Abundance. Although the primary aim of this study was to determine whether the theophylline responsive riboswitch works in Clostridia, we also attempted to further characterize certain aspects of riboregulator function. It is well-known that the RNA structure plays an important role in controlling both mRNA transcription $^{31}$ and protein synthesis. ${ }^{32,33}$ It follows that the response of the theophylline responsive riboswitch can be altered, or even abolished, by modifying the $5^{\prime} \mathrm{UTR}^{27}$ This also implies that the performance of a given riboswitch will inevitably be dependent on the genetic context. Therefore, we sought to determine whether the differences in inducible CAT expression observed when using different $5^{\prime}$ UTRs were solely due to the abundance of mRNA transcripts. To this end, mRNA transcribed under the control of riboswitch-G located downstream of either $\mathrm{P}_{f d x}$ or $\mathrm{P}_{f d x} *\left(\mathrm{P}_{f d x}\right.$ along with the native $5^{\prime}$ UTR sequence) was quantified via RT-qPCR.

Results showed a 2.5-fold increase in the mRNA transcribed from $\mathrm{P}_{f d x} *$ compared with the mRNA transcribed from $\mathrm{P}_{f d x}$ in cultures induced with $2 \mathrm{mM}$ theophylline (Figure 4). It should

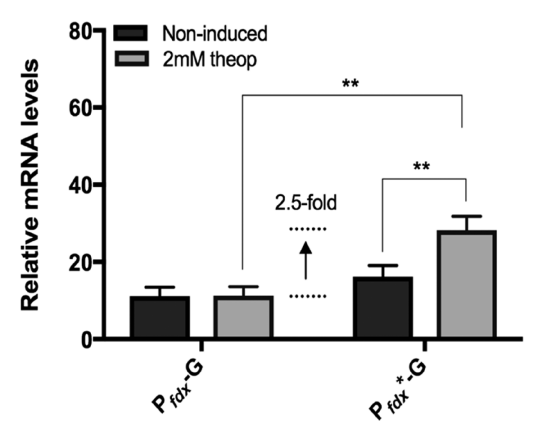

Figure 4. $\mathrm{P}_{f d x}-\mathrm{G}$ and $\mathrm{P}_{f d x *}-\mathrm{G}$ transcript abundance comparison. Transcript abundance determined by RT-qPCR relative to two reference genes, $16 \mathrm{Srrn}$ and gyrA. Total RNA was isolated from late exponential cultures grown in the presence and absence of $2 \mathrm{mM}$ theophylline. Error bars represent standard deviations of three biological replicates. Asterisks indicate statistically significant induction values (paired/unpaired two-tailed Student's $t$-test).

be noted that this increase in mRNA levels was accompanied by a 2.6-fold increase in CAT expression (Figure 3b), as summarized in Table S6, which suggests a direct link between the relative abundance of catP transcripts and CAT expression. The levels of mRNA when $\mathrm{P}_{f d x}$ is placed upstream of the riboswitch without its native $5^{\prime}$ UTR were similar in the absence or presence of the inducer. Interestingly, there was a significant difference in the amount of transcript between the on/off state in the case of $\mathrm{P}_{f d x}{ }^{*}$, abundance being higher when the inducer was added. These differences reflect the role the sequence downstream of the TSS plays in mRNA abundance, possibly as a result of a complex interplay between the processes of transcription, translation and mRNA degradation. Plausible explanations could include that the additional nucleotides downstream of the TSS create an RNA conformation that is less susceptible to degradation or that the amount of transcript is indirectly influenced by the conformation of the mRNA (i.e., with or without theophylline) and its associated translation efficiency. ${ }^{32}$

Altogether, these data indicate that the studied riboswitch responds to theophylline regardless of the genetic context and that the appropriate combination of a riboswitch with a certain promoter and the characterized 5' UTRs would, therefore, allow for the adjustment of a desired regulatory window.

Functional Characterization of Theophylline-Riboswitches in Clostridium. To assess the dose dependency of riboswitch regulation, strains harboring plasmids that carried the riboswitch-G downstream of $\mathrm{P}_{f d x}$ or $\mathrm{P}_{f d x}$ * were cultivated with increasing concentrations of theophylline, ranging from 0.1 to $10 \mathrm{mM}$ (Figure 5a). Increasing doses of theophylline would be expected to result in higher levels of gene expression. Concentrations higher than $5 \mathrm{mM}$, however, resulted in a reduction of growth, in agreement with previously reported results in other Gram-positive and Gram-negative bacteria ${ }^{34}$ (Figure $5 b$ ). The translational switches were also analyzed for CAT expression over time in the presence and absence of 2 $\mathrm{mM}$ theophylline. As shown in Figure 5c, CAT expression above the level of the uninduced culture was already detectable $30 \mathrm{~min}$ after induction ( $4.5 \mathrm{~h}$ of growth). The maximum level of reporter gene expression was reached at 8 and $10 \mathrm{~h}$ when using $\mathrm{P}_{f d x}$ and $\mathrm{P}_{f d x}{ }^{*}$, respectively. Although both have a similar profile of induction, after $24 \mathrm{~h}$, CAT expression was reduced by $75 \%$ in $\mathrm{P}_{f d x}$, whereas only by $40 \%$ in $\mathrm{P}_{f d x} *$. Given that in both cases the translated product is the same (CAT), these results suggest that the reversal of induction is strongly dependent on the abundance or stability of the mRNA.

The most effective inducers are nonmetabolizable. To establish whether this was the case in C. sporogenes, cells were cultivated in TYG medium supplemented with $2 \mathrm{mM}$ theophylline. The concentration of theophylline was monitored by HPLC-MS analysis of cell-free supernatant samples over time. Results showed that the concentration of theophylline remained constant over the course of the experiment, with a slight and nonsignificant increase in the culture supernatant $20 \mathrm{~h}$ after induction. As a result of the manual processing of several samples and the inevitable delay between culture induction and sample harvesting, it is plausible that a small quantity of theophylline could have already been uptaken by the cells at T0. Some of this intracellular theophylline may have been released in the medium following cell lysis at $20 \mathrm{~h}$, thus accounting for the small increase of extracellular inducer concentration observed at this time point (Figure 5d).

RiboCas: A Highly Efficient CRISPR/Cas9-Based Tool for Clostridium. With a tightly inducible gene expression system for Clostridium to hand, we explored the possibility of generating an efficient clostridial genome editing tool based on CRISPR/Cas9. Figure 6 illustrates the general RiboCas-based editing process and the assembly of the RiboCas vector series, generically named $\mathrm{pRXCasN}$ vectors, where $\mathrm{X}$ and $\mathrm{N}$ refer to the riboswitch and the promoter driving the sgRNA, respectively.

It contains the four unique restriction sites AscI, FseI, PmeI, and Sbfl, which are used for modular assembly. (-) is the Gram-negative origin of replication that allows replication of 



Figure 5. Characterization of theophylline riboswitches in C. sporogenes. Dynamic and kinetic profiles of the theophylline responsive riboswitch located downstream of $\mathrm{P}_{f d x}$ or $\mathrm{P}_{f d x} *$. (a) Response to different concentrations of the inducer theophylline. Cells containing the reporter plasmid with riboswitch-G downstream of either $\mathrm{P}_{f d x}$ or $\mathrm{P}_{f d x}$ * were cultivated in TYG medium supplemented with various concentrations of theophylline (0, $0.1,0.5,2,5$, and $10 \mathrm{mM}$ ) at early exponential growth phase $\left(4 \mathrm{~h}\right.$ of growth, $\left.\mathrm{OD}_{600} \approx 0.5\right)$; CAT activity was measured on cell lysates from stationary cultures. (b) Optical density $\left(\mathrm{OD}_{600}\right)$ of $C$. sporogenes harboring the same reporter plasmids in response to $0.1-10 \mathrm{mM}$ of theophylline supplemented at time zero. (c) CAT expression profiles over time. Cells harboring the reporter plasmids were cultivated in TYG media in the absence or presence of $2 \mathrm{mM}$ theophylline; CAT was measured on cell lysates from stationary cultures. (d) Stability profile of theophylline in C. sporogenes. In all cases, error bars represent the standard deviations of three biological replicates.

the shuttle plasmid in E. coli. (+) is the Gram-positive replicon. The application-specific module harbors the components of the editing tool, including the gene encoding Cas9, the sgRNA and the homologous DNA template needed for homologous recombination. The terminators CD0164 (derived from C. difficile) and $\mathrm{T}_{f d x}$ (derived from the C. pasteurianum ferredoxin gene) are placed downstream of cas 9 and the sgRNA, respectively. The system was designed to be 
a
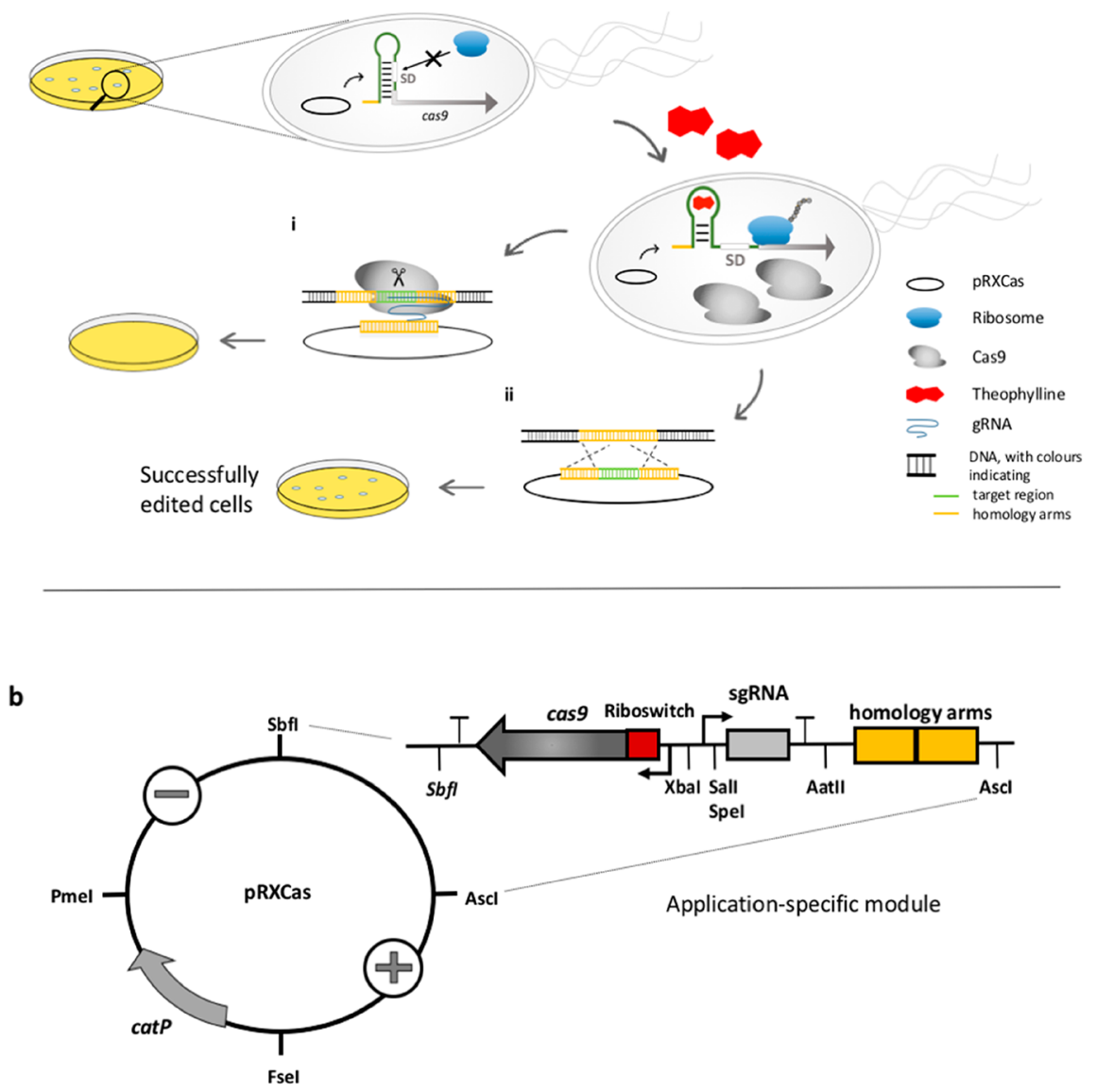

Figure 6. pRXCas vector series and RiboCas functionality. (a) Illustration of the RiboCas-mediated genome editing. Transformed cells survive on selective media in the absence of theophylline due to the tight repression exerted by the riboswitch, which impedes the translation of the nuclease Cas9. After induction, the translated Cas9 forms a complex with the sgRNA (Cas9-sgRNA) inducing a double-strand breakage on the target DNA. (i) The Cas9-sgRNA complex is lethal to the unedited cells, which are killed during the process. (ii) Cells are able to survive only if homologous recombination occurs between the gene-targeting plasmid and the genome. (b) Schematic illustration of the RiboCas vector.

compatible with our previous pMTL80000 vector series enabling rapid exchange of selection markers and origins of replication. $^{30}$

In order to have an appropriate control over cas 9 expression, both in the uninduced state and after induction, we selected two riboswitches, riboswitches $-\mathrm{E}$ and $-\mathrm{G}$, from our riboswitch library, both located downstream of the promoter $\mathrm{P}_{f d x}$ (Figure 2a). Riboswitch-E had shown the tightest repression in the noninduced state among all tested riboswitches; on the other hand, riboswitch-G showed higher background levels but increased expression when the inducer was added. Two promoters, the constitutive promoter of the Clostridium acetobutylicum araE gene $\left(\mathrm{P}_{\text {araE }}\right.$, associated with the protein coding gene Ca_1339) and the synthetic promoter J23119, were chosen to bring about the expression of the sgRNA. Both had been previously used for CRISPR strategies with Clostridium species. $^{21,35}$ In all cases, the editing template comprised two homology arms composed of the regions of the chromosome needed to replace the target region. As a proofof-principle, we decided to target the spoIIR gene (Clspo_c01510, encoding the stage II sporulation protein R). The DNA modules encoding either riboswitch-E or -G, cas9, the sgRNA downstream of either $\mathrm{P}_{\text {araE }}$ or J23113 and the DNA editing template were inserted into the $\mathrm{pCB} 102$-based modular vector pMTL83151, ${ }^{30}$ yielding the following $\mathrm{pRXCasN}$ vector series: pRECas1-IIR, pRECas2-IIR, pRGCas1-IIR, and
pRGCas2-IIR (Table S5). The same vectors lacking the editing homology arms were assembled to determine the killing capacity of Cas9, named pRECas1G-IIR, pRECas2GIIR, pRGCas1G-IIR, and pRGCas2G-IIR. Two vectors, pRECasC and pRGCasC, lacking both the homology arms and sgRNA, were also created as a control for conjugation efficiency comparisons.

Transfer of the resulting vectors into $C$. sporogenes was performed via conjugative plasmid transfer from an E. coli donor, plating the conjugated cultures onto media supplemented with thiamphenicol in the presence and absence of 5 $\mathrm{mM}$ theophylline. As expected, the different plasmid constructs were transferred with different conjugation efficiencies (Figure $7 \mathrm{a})$. The combination of riboswitch-E and the promoter $\mathrm{P}_{\text {araE }}$ upstream of the sgRNA (pRECas1) provided the highest conjugation efficiency $\left(1.21 \times 10^{-7} \mathrm{CFU} /\right.$ donor; CFU: colony forming units), comparable to the $1.46 \times 10^{-7} \mathrm{CFU} /$ donor obtained with pRECasC (nontargeting Cas9). Conjugative transfer of the control plasmids, pRECasC and pRGCasC, resulted in similar colony counts on plates with and without theophylline (Figure $7 \mathrm{~b}$ ), indicating a very low off-target activity in the absence of the targeting module. In all cases the conjugation efficiency was lower if riboswitch-G regulated the expression of Cas9, which implies a higher background expression level in the absence of the inducer and agrees with our previous CAT results. No colonies were observed on 

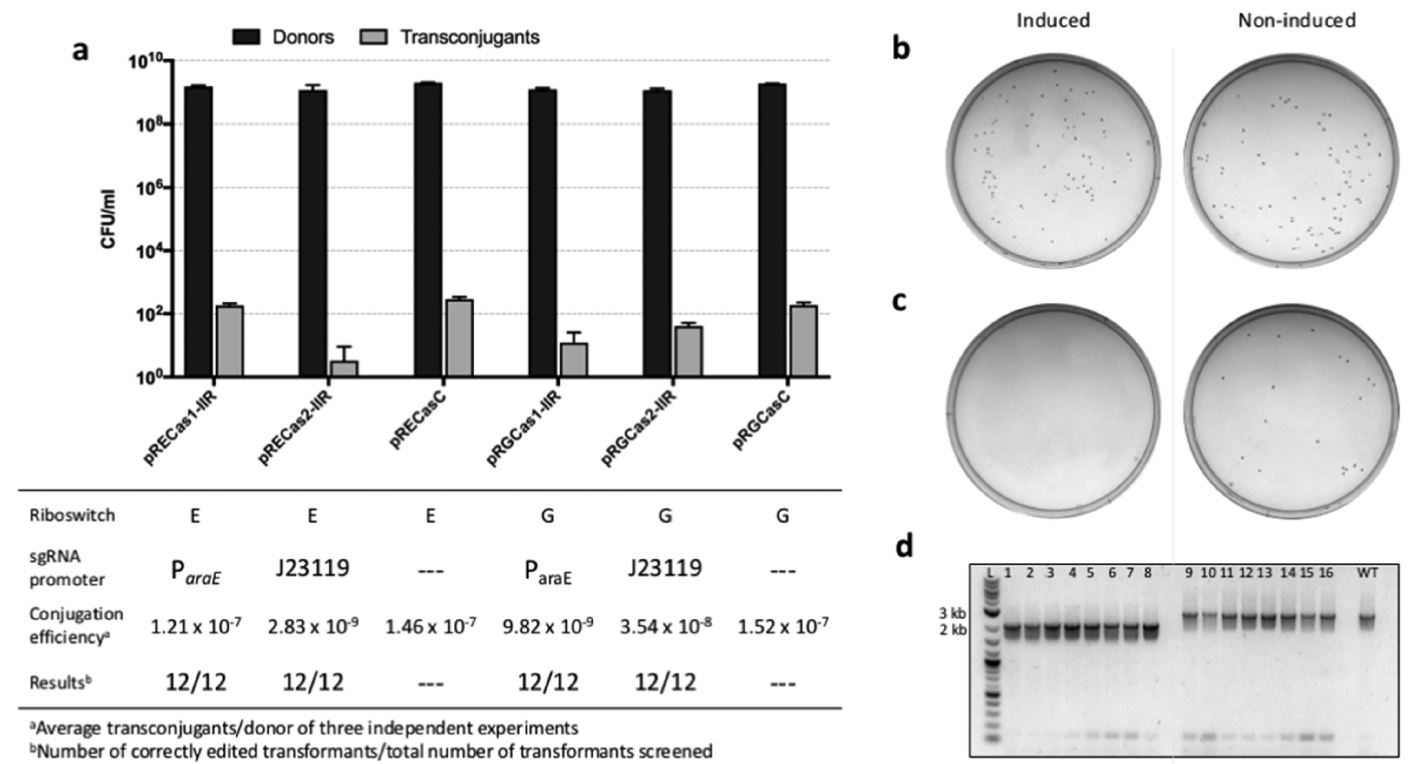

Figure 7. RiboCas efficiency. (a) Conjugation efficiency of different RiboCas plasmids involved in CRISPR-mediated gene editing. Error bars represent standard deviations of three biological replicates on three independent experiments. The data summarize the obtained editing results from each RiboCas plasmid. (b) CFU obtained from conjugation of pRECas1C (RiboCas vector lacking both homology arms and sgRNA) on selective media in the presence and absence of the inducer theophylline. (c) CFU obtained from conjugation of pRECas1G-IIR (RiboCas vector lacking the homology arms) on selective media in the presence and absence of theophylline. (d) Confirmation of the gene deletion using colony PCR. Lane L, the 2-log DNA marker with sizes $(\mathrm{kbp})$ on the left; lanes 1-8, colony PCR on colonies from selective plates containing theophylline; lanes 9-16, colony PCR on colonies from selective plates lacking theophylline; WT (wildtype).

a
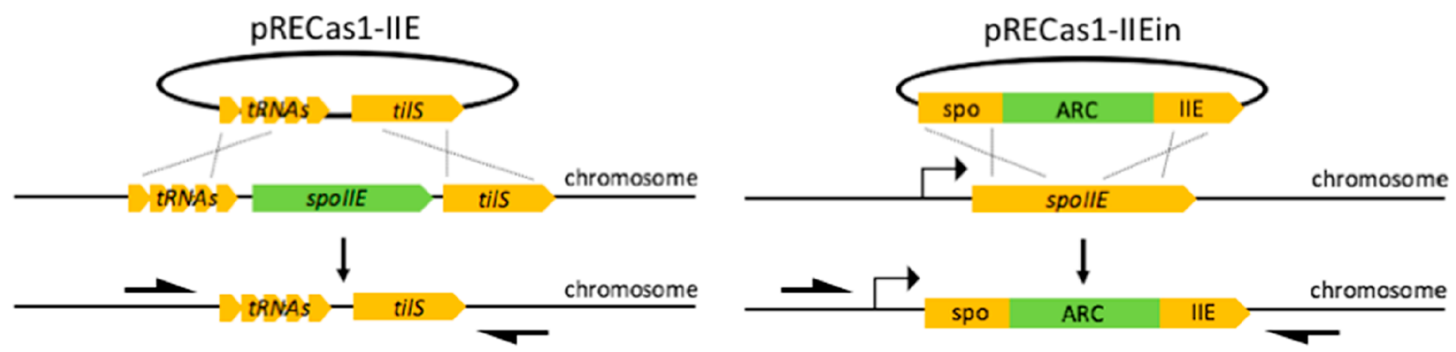

b

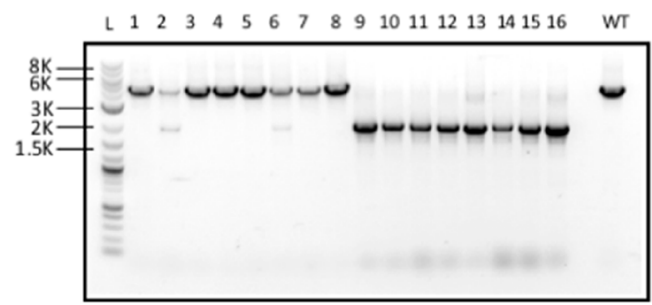

WT $4.3 \mathrm{kbp}$

L $1 \begin{array}{llllllllllllll} & 2 & 4 & 5 & 6 & 7 & 8 & 9 & 1011 & 12 & 13 & 141516 \quad \text { WT }\end{array}$

MUT $1.9 \mathrm{kbp}$

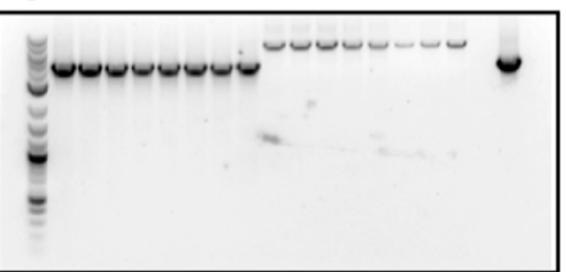

MUT $7.2 \mathrm{kbp}$

WT $4.3 \mathrm{kbp}$

Figure 8. Deletion and insertion of DNA into the chromosome of C. sporogenes using RiboCas. (a) Schematic representation of the deletion of spoIIE (via pRECas1-IIE) and the integration of an ARC in the spoIIE locus (via pRECas1-IIEin) in the chromosome of C. sporogenes. The ARC comprises the $\mathrm{ermB}$ gene that confers resistance to the antibiotic erythromycin and the sequence encoding the reporter enzyme $\beta$-glucuronidase from E. coli. (b) PCR screening of the spoIIE deletion and integration of the ARC. The 1.9, 7.2, and $4.3 \mathrm{kbp}$ bands represent the spoIIE deletion, ARC integration and WT genotypes, respectively. Lane L, 2-log DNA marker with sizes (kbp) on the left; lanes 1-8 uninduced colonies; lanes 916 induced colonies.

plates supplemented with theophylline if the sgRNA was expressed, whether or not the editing template was included, indicating highly efficient Cas9-mediating killing of host cells (Figure 7c). These results also suggest that it could be possible to select mutant cells just after transformation if the homologous recombination event had occurred during the recovery time.
Individual colonies harboring the different CRISPR/Cas9 vectors and the DNA editing template were picked and transferred to selective plates in the presence of theophylline. In all cases, amplicons of $\sim 2.2 \mathrm{kbp}$ instead of $\sim 2.9 \mathrm{kbp}$ size were detected after screening, implying the expected $\sim 0.7 \mathrm{kbp}$ deletion of spoIIR and an editing efficiency of $100 \%$ (Figure $7 d$ ). Desired deletions were confirmed by sequencing the PCR products. Screening of uninduced colonies generated the 
amplicon of the WT strain $(2.9 \mathrm{kbp})$; sequencing of these PCR products did not show insertions/deletions. These results suggest that the dynamic range of riboswitch-E is appropriate for a Cas9-base editing system, providing both undetectable off-target activity and high selection capacity. The consistency of these results was confirmed by three independent experiments, verifying the robustness of the RiboCas system and its suitability for more ambitious genome editing strategies.

Application of RiboCas for the Deletion and Integration of Larger Fragments. To further illustrate the versatility of the RiboCas system we attempted to both delete and integrate larger fragments into the genome of C. sporogenes. The $2.4 \mathrm{kbp}$ spoIIE gene (Clspo_c37040) was selected as the target locus. Two sets of homology arms were designed to either delete the target sequence or to integrate a 3 $\mathrm{kbp}$ cassette that encompasses a gene (ermB) that confers resistance to erythromycin and the gene encoding the reporter $\beta$-glucuronidase (gusA) from E. coli, together forming a large, innocuous DNA cargo (Figure 8a). The editing templates and the sgRNA sequences targeting spoIIE were cloned into pRECas1 (the most efficient RiboCas system in C. sporogenes in terms of conjugation efficiency) generating pRECas1-IIE (deletion vector) and pRECas1_IIEin (integration vector). Both vectors were successfully conjugated into $C$. sporogenes. After conjugation, induction of cas 9 expression was performed by restreaking individual colonies harboring pRECas1-IIE or pRECas1-IIEin onto plates supplemented with thiamphenicol and $5 \mathrm{mM}$ theophylline. PCR was performed on uninduced cultures and on colonies that had grown for $24 \mathrm{~h}$ in the presence of the inducer. All of the screened colonies were confirmed as positive mutants for spoIIE deletion and integration of the antibiotic resistance cassette (ARC) (Figure $8 \mathrm{~b}$ ). Generally, PCR products obtained from colonies grown in the absence of the inducer corresponded to unedited cells, demonstrating the tightness of RiboCas system in the absence of the inducer. Only a few colonies $(<10 \%)$ were composed of mixtures of mutant and WT cells. Finally, to validate the efficiency and detect off-target events that could have arisen during the editing process, the genome of six independent mutants of $C$. sporogenes was sequenced. In all of them, no modifications apart from the intended editions were detected when compared to the resequenced $C$. sporogenes parent strain.

RiboCas Demonstrates Universality in Clostridium. To determine the applicability of the theophylline responsive riboswitches and the RiboCas editing tool in other clostridial species we first sought to delete the same gene, spoIIE, in the solventogenic species C. pasteurianum and in the pathogens C. difficile and C. botulinum. Vectors pRECas1, containing the homology arms and the sgRNA to target the spoIIE gene in each species were introduced into the host organisms C. pasteurianum DSM 525- $\mathrm{H}^{6}$, C. difficile 630 and the group I C. botulinum strain ATCC 3502. C. pasteurianum was transformed via electroporation, whereas plasmids were introduced into $C$. difficile and $C$. botulinum by conjugative plasmid transfer from an E. coli donor. Simultaneously, the control plasmid pRECas1C (RiboCas vector lacking both homology arms and sgRNA), was also introduced into the different hosts to determine the leakiness of the system and its impact on the transformation/conjugation process. All transformations were plated on media supplemented with thiamphenicol, both in the presence and absence of theophylline. The transformation frequency of the targeting RiboCas vector in C. pasteurianum was only $20 \%$ lower than that of the control vector $(5000 \mathrm{CFU} / \mu \mathrm{g}$ DNA in pRECas1 vs 6500 $\mathrm{CFU} / \mu \mathrm{g}$ DNA in pRECas1C) (Figure 9). On average a

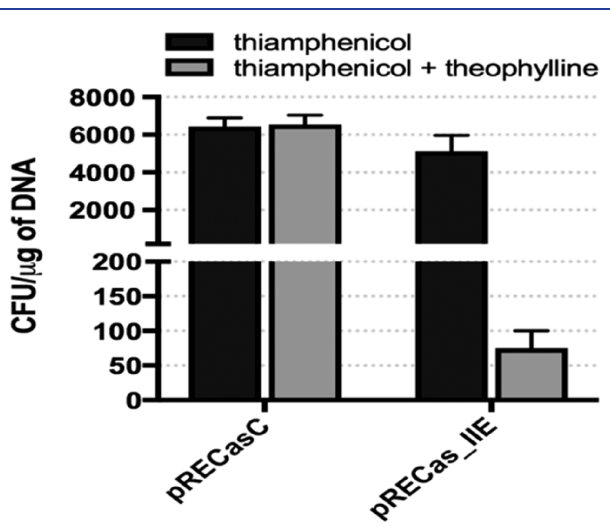

Figure 9. Transformation efficiency of RiboCas into C. pasteurianum. Transformation efficiencies of RiboCas plasmids in C. pasteurianum, without (pRECasC) or with (pRECas-IIE) the spoIIE-targeting sgRNA and the DNA editing template, when plated on selective media in the absence and presence of the inducer theophylline.

transformation efficiency of $75 \mathrm{CFU} / \mu \mathrm{g}$ DNA was obtained on plates containing theophylline, demonstrating that the theophylline responsive riboregulator is functional in C. pasteurianum. PCR screening of these colonies confirmed the mutant genotype of the edited cells without the need of further restreaking; the unedited phenotype was maintained on plates lacking the inducer. Colonies from noninduced plates were streaked onto plates containing both thiamphenicol and theophylline and colony PCR used to confirm their identity as deletion mutants in 24 out of the 24 colonies screened (100\% efficiency) (Table 1). A similar transformation pattern was found when conjugating the RiboCas vectors into C. difficile and C. botulinum (data not shown), validating the induction ability of riboswitches and the efficiency of the RiboCas tool in both organisms. Screening of $C$. difficile colonies after induction confirmed an efficiency of $100 \%$ while maintaining the WT phenotype on colonies not exposed to theophylline (Table 1). Interestingly, and in contrast to the results observed in other clostridial species, C. botulinum colonies on plates lacking theophylline were composed of either mutants or a mixture of mutant and WT populations. This may suggest that although inducible, riboswitches exhibit a higher background expression in C. botulinum but that the selective capacity of Cas9 still allows the selection of only edited cells.

A summary of the editing attempts carried out in this study, including the protospacer sequence used, are summarized in Table 1. It is worth noting that, despite pRECas1 being the most efficient in this study (in terms of conjugation efficiency), vectors incorporating the promoter J23119 might be suitable for organisms where $\mathrm{P}_{\text {araE }}$ is expected to be natively repressed (i.e., in C. acetobutylicum).

\section{DISCUSSION}

This study shows the first implementation of synthetic riboswitches in the genus Clostridium and demonstrates their application in highly efficient genome editing. Previously existing as well as newly designed theophylline-dependent riboswitches were functional in C. sporogenes, with a response 
Table 1. Genome Editing Results in Different Clostridium Species

\begin{tabular}{|c|c|c|c|c|}
\hline strain & target & deletion (kpb) & protospacer sequence & efficiency $^{a}$ \\
\hline C. pasteurianum DSM 525 & spoIIE & 2.4 & ggaaactcctaaatatcacg & 100 \\
\hline C. difficile 630 & spoIIE & 2.4 & ggaattttagatttaaaacg & 100 \\
\hline C. botulinum ATCC 3502 & spoIIE & 2.4 & ttttataaagcgaggaactg & 100 \\
\hline C. sporogenes NCBI 10696 & spoIIE & 2.4 & ttaattataggatatgtaaa & 100 \\
\hline
\end{tabular}

that is dependent on inducer concentration. In particular, the novel riboswitch-G outperformed previously published riboswitches, exhibiting a large dynamic range and very low basal expression. By replacing the promoter and modifying the $5^{\prime}$ UTR sequence located upstream of the riboswitch, we generated a library of inducible switches with a full set of dynamic ranges, suitable for applications where high levels of gene expression are needed as well as for the expression of detrimental proteins. In all cases, the riboswitch was able to respond to the inducer theophylline, ensuring its performance independent of the genetic context.

The usefulness of these tight and versatile riboregulators was demonstrated by generating a novel riboswitch-based CRISPR editing tool-RiboCas-that overcomes the main obstacles associated with Cas9 editing tools, including the very low transformation efficiencies and the inability to select only edited cells as a result of excessive Cas9 toxicity. ${ }^{36,37}$ We attribute the high efficiency of RiboCas to several reasons. First, the theophylline responsive riboswitch can be designed to have the desired regulatory window, whereby there should be very low basal expression of Cas9 in the absence of inducer, allowing homologous recombination to occur before Cas9 mediates the site-specific double strand break, and after induction, to be expressed at a level that is sufficient for effective genome DNA cleavage but which minimizes toxicity that can be associated with Cas9 overexpression. Second, riboswitches are smaller structures compared to systems that require a transcription factor; in a riboswitch-based system, 84 nucleotides are enough to tightly control the expression of Cas9, minimizing the size of the plasmid employed and enabling higher transformation efficiencies. Third, because repression occurs at the level of translation, the RiboCas system allows the use of high-copy origins of replication, avoiding the undesired effects linked to read-through transcription on the plasmid backbone and facilitating the processes of cloning, screening, and sequencing. Finally, and due to the aforementioned characteristics, RiboCas permits the confinement of all the essential components of a functional genome editing tool to the same plasmid.

The universal nature of RiboCas was demonstrated through its successful application in several clostridial species, including the nonpathogen $C$. sporogenes, the solventogenic C. pasteurianum, and the pathogens $C$. difficile and C. botulinum. The derivation and application in C. sporogenes of a CRISPR-Cas9based system was recently reported. ${ }^{38}$ In contrast to our work, mutants could be only obtained when the components of the system were split between two separate vectors, one containing the sgRNA and editing template and the other carrying cas9. One consequence was that the conjugative transfer of the second vector was only possible after $72 \mathrm{~h}$ of donor/acceptor cocultivation; generally, the time required to transfer the multicomponent RiboCas vector into C. sporogenes is $8 \mathrm{~h}$. Previous work had also enabled the use of Cas9 for editing purposes in C. pasteurianum; ${ }^{13,14}$ however, the strategies employed suffered from very low transformation efficiencies that might compromise more ambitious applications. This is also the case in $C$. difficile, ${ }^{17,18}$ where the performance of CRISPR has been suboptimal. In particular, conjugation of CRISPR plasmids in this organism has yielded invariably low efficiencies. This is likely due to the high toxicity associated with the Cas9 endonuclease activity. The recent application of a CRISPR-Cpf1 editing tool enabled the deletion of various genes with high efficiency. ${ }^{39}$ However, because Cpf1 nuclease recognizes a T-rich PAM sequence $\left(5^{\prime}-\mathrm{TTTN}-3^{\prime}\right)$, we hypothesize that off-target events across T-rich genomes (such as those from species belonging to the genus Clostridium) are more likely to occur if compared with Cas9, which recognizes the PAM sequence $5^{\prime}-\mathrm{GGN}-3^{\prime} .{ }^{40}$ To our knowledge, this is the first report of CRISPR targeted gene disruption in a C. botulinum chromosome.

As the field of synthetic biology progresses toward more practical applications, the technologies that have been developed and optimized for model prokaryotes need to be extended to more exotic chassis. This study provides a new set of tools that facilitate the efficient manipulation of clostridial pathogens as well as those Clostridium species of industrial and therapeutic relevance. As riboswitches are RNA-based devices that control gene expression without the need of protein factors, we anticipate that RiboCas will function in bacterial chassis other than Clostridium and in a wide range of applications for which tight control of gene expression is required.

\section{MATERIALS AND METHODS}

Strains, Media, and Growth Conditions. All the E. coli and Clostridium strains used in this study are listed in Table S2. E. coli TOP10 (Invitrogen) was used as a general host for plasmid construction and propagation. E. coli CA434 was used as the donor strain for conjugation. Plasmid DNA for the transformation of $C$. pasteurianum was methylated in vivo by propagation in the E. coli host CR1, which harbors the plasmid pCR1, encoding the M.BepI methyltransferase, as previously described. $^{6}$ All E. coli strains were transformed through electroporation using a MicroPulser system (BioRad). E. coli strains were grown at 30 or $37{ }^{\circ} \mathrm{C}$, in Luria-Bertani (LB) medium supplemented with chloramphenicol $(25 \mu \mathrm{g} / \mathrm{mL}$ in solid and $12.5 \mu \mathrm{g} / \mathrm{mL}$ in liquid media), erythromycin $(500 \mu \mathrm{g} /$ $\mathrm{mL})$ or kanamycin $(50 \mu \mathrm{g} / \mathrm{mL})$ when necessary. Growth media for the different Clostridium species are specified on Table S3. Media for clostridial strains were supplemented with the following antibiotic/inducer/supplement when appropriate: thiamphenicol $(15 \mu \mathrm{g} / \mathrm{mL})$, erythromycin $(10 \mu \mathrm{g} / \mathrm{mL})$, cefotoxin $(16 \mu \mathrm{g} / \mathrm{mL}), \mathrm{D}$-cycloserine $(500 \mu \mathrm{g} / \mathrm{mL})$, theophylline $(0.1-10 \mathrm{mM})$, glucose $0.05 \% \mathrm{w} / \mathrm{v}$. Clostridium strains were grown at $37{ }^{\circ} \mathrm{C}$ in an anaerobic cabinet (MG1000 anaerobic workstation; Don Whitley Scientific Ltd.).

Reagents. All PCR reactions were performed using KODHot Start Polymerase 2X Master Mix (Merck Millipore) or 
DreamTaq Green PCR Master Mix (Thermo Fisher Scientific). T4 ligase (Promega) was used for DNA ligation reactions. Restriction enzymes were purchased from New England Biolabs. Theophylline was purchased from SigmaAldrich.

Plasmid Design, Construction, and Transformation. Oligonucleotide primers were synthesized by Sigma-Aldrich and are listed in Table S4. Plasmids were constructed by restriction enzyme-based cloning procedures. ${ }^{41}$ Constructs were verified by DNA sequencing (Eurofins). All the plasmids used in this study are listed in Table S5. Details of plasmid construction are given in the Supporting Information. Protospacer sequences were designed according to the protocol described at http://benchling.com/pub/ellis-crsprtools.

Growth and CAT Activity Assays. For each tested system, three independently conjugated C. sporogenes cultures were grown for $12 \mathrm{~h}$ with selection before being diluted to a starting $\mathrm{OD}_{600}$ of 0.01 in fresh medium. For evaluation of CAT activity at a single time point, cultures were induced at an $\mathrm{OD}_{600} \approx 0.5$ with $2 \mathrm{mM}$ theophylline; cultures were collected at stationary phase $4 \mathrm{~h}$ after induction. For CAT expression assays over time, cultures were prepared as for the single time point measurements, collecting the samples at the specific data points. For dose-dependency assays, cultures were induced with increasing concentrations of theophylline $(0-10 \mathrm{mM})$. In all cases, after sample collection, pellets were obtained and stored at $-20{ }^{\circ} \mathrm{C}$ until CAT activity was determined.

CAT activity was measured on cell lysates according to the method of Shaw. ${ }^{42}$ Cell lysates were obtained using BugBuster Master Mix lysis buffer (Novagen), according to the manufacturer's protocol. A $150 \mu \mathrm{L}$ aliquot of a master mix, consisting of $94 \mathrm{mM}$ Tris buffer ( $\mathrm{pH} 7.8), 0.19 \mathrm{mM}$ acetyl Coenzyme A, $0.0833 \mathrm{mM}$ DTNB (5,5'-dithiobis-2-nitrobenzoic acid), and $0.005 \%(\mathrm{w} / \mathrm{v})$ chloramphenicol, was injected into each well of a 96-well clear-bottom plate (Greiner Bio One International) containing $10 \mu \mathrm{L}$ of cell lysates and 40 $\mu \mathrm{L}$ of $100 \mathrm{mM}$ Tris buffer ( $\mathrm{pH}$ 7.8). Absorbance was measured at $412 \mathrm{~nm}$ for $1 \mathrm{~min}$ using a CLARIOstar plate reader (MBG Labtech $\mathrm{GmbH}$ ), set at $25{ }^{\circ} \mathrm{C}$. The rate of increase of absorption was used to calculate CAT activity $(\mathrm{U} / \mathrm{mL})$ using the following equation, where 0.2 is the total volume (in $\mathrm{mL}$ ) of assay, $d f$ is the dilution factor, 0.0136 is the micromolar extinction coefficient for DTNB at $412 \mathrm{~nm},{ }^{43}$ and 0.01 is the volume of cell lysate used.

$$
\begin{aligned}
& \text { Units } / \mathrm{mL} \text { CAT } \\
& =\frac{\left.\left(\Delta A_{412} / \mathrm{min} \text { test }-\Delta A_{412} / \min \text { blank }\right)(0.2)(d f)\right)}{(0.0136)(0.01)}
\end{aligned}
$$

CAT activity was further normalized by the total protein concentrations obtained using a BCA assay (Thermo Scientific).

For growth experiments, precultures grown for $12 \mathrm{~h}$ were diluted to a starting $\mathrm{OD}_{600}$ of 0.01 with fresh medium containing different concentrations of the inducer theophylline $(0-10 \mathrm{mM})$. Because theophylline is dissolved in DMSO, appropriate quantities of DMSO were added to all the cultures to account for any effect DMSO might have on growth.

HPLC-MS Analysis. To analyze if $C$. sporogenes consumes theophylline, $12 \mathrm{~h}$ cultures were diluted to a staring $\mathrm{OD}_{600}$ of 0.01 . At an $\mathrm{OD}_{600}$ of $\approx 0.5$ cultures were induced with $2 \mathrm{mM}$ theophylline. Sample volumes of $1 \mathrm{~mL}$ samples were taken immediately and then at $2,4,6,8,10$, and $20 \mathrm{~h}$ after supplementation of theophylline. Samples were centrifuged for $10 \mathrm{~min}$ and $10000 \mathrm{~g}$. Supernatants were used to determine the concentration of theophylline using an Alliance HT 2795 HPLC (Waters corporation) coupled to a Micromass Quattro LC Mass Spectrometer (Waters Wilmslow) in positive mode from $\mathrm{m} / z 70$ to 500 for all samples at a scan rate of $1 \mathrm{cycle} / \mathrm{s}$ and equipped with an electrospray source. The general parameters were as follows: capillary voltage $2000 \mathrm{~V}$, sampling cone $30 \mathrm{~V}$, source temperature $130{ }^{\circ} \mathrm{C}$, desolvation temperature $350{ }^{\circ} \mathrm{C}$, cone gas flow $64 \mathrm{~L} / \mathrm{h}$, and desolvation gas flow $621 \mathrm{~L} / \mathrm{h}$.

Prior to ionization, chromatographic separation was achieved using a Supelco Ascentis Express HPLC column (100 mm $\times 3 \mathrm{~mm}, 2.7 \mu \mathrm{M}$, Sigma-Aldrich). The mobile phase consisted of (A) water with $0.1 \% \mathrm{v} / \mathrm{v}$ formic acid and (B) methanol with $0.1 \% \mathrm{v} / \mathrm{v}$ formic acid. In all HPLC runs the elution gradient started at $95 \% \mathrm{~A}, 5 \% \mathrm{~B}$ increasing to $10 \% \mathrm{~A}$, $90 \%$ B, followed by a 5 min re-equilibration period. A sample volume of $10 \mu \mathrm{L}$ was injected for each HPLC run. The column was operated at $40{ }^{\circ} \mathrm{C}$ with a flow rate of $0.4 \mathrm{~mL} / \mathrm{min}$. The HPLC run contained blanks and the sample-relevant standard solution. Samples and standards were filtered using a $0.2 \mu \mathrm{M}$ filter.

Conjugation/Transformation Efficiency Determination and Mutant Screening. For conjugation efficiency assays, the donor strain, E. coli CA434 (in triplicates), was grown overnight at $30{ }^{\circ} \mathrm{C}$ in $\mathrm{LB}$ supplemented with kanamycin and chloramphenicol. For each replica, two $1 \mathrm{~mL}$ cultures were centrifuged at $6000 \mathrm{~g}$ for $1 \mathrm{~min}$ and then washed once with phosphate-buffered saline (PBS). After a second centrifugation step, one of the two cultures was used to quantify the donor by plating the appropriate serial dilutions onto LB plates. The second culture was transferred to the anaerobic cabinet and mixed with $200 \mu \mathrm{L}$ of a $12-\mathrm{h} \mathrm{C}$. sporogenes culture. The mixture was spotted onto the TYG medium supplemented with glucose. After $8 \mathrm{~h}$, cells were harvested, resuspended in 500 $\mu \mathrm{L}$ of PBS and plated onto media supplemented with thiamphenicol and D-cycloserine, in the presence and absence of the inducer theophylline. After 24-48 h of incubation at 37 ${ }^{\circ} \mathrm{C}$, colonies were counted and conjugation efficiency was calculated as total transconjugants per $1 \mathrm{~mL}$ of donor strain.

Transformation efficiency in $C$. pasteurianum was determined as the average of three independent transformations with $4 \mu \mathrm{g}$ of plasmid DNA.

Single colonies were picked randomly and screened for desired mutants using colony PCR and specific flanking primers. In all cases, mutations were further confirmed with Sanger sequencing (data not shown).

DNA Sequencing and Bioinformatics. Genomic DNA was isolated from $C$. sporogenes overnight cultures using phenol-chloroform extraction. Genome sequencing was provided by MicrobesNG, using their Illumina-based, Standard Whole-Genome Service (http://www.microbesng.uk). Whole genome sequencing data were assembled and analyzed using the CLC Genomics Workbench version 10 (Qiagen). Resequencing analysis for the identification of single nucleotide variants (SNVs) was carried out using the Basic Variant Detection tool and the program's default parameters. 


\section{ASSOCIATED CONTENT}

\section{S Supporting Information}

The Supporting Information is available free of charge on the ACS Publications website at DOI: 10.1021/acssynbio.9b00075.

Further experimental details and additional tables and figures described in the main text (PDF)

\section{AUTHOR INFORMATION}

\section{Corresponding Author}

*E-mail: nigel.minton@nottingham.ac.uk.

\section{ORCID}

Nigel P. Minton: 0000-0002-9277-1261

\section{Author Contributions}

I.C. designed the study; I.C., D.G., M.Z., R.R. performed the experiments; I.C. and N.P.M. analyzed the data; and all authors contributed to the manuscript.

\section{Notes}

The authors declare no competing financial interest.

\section{ACKNOWLEDGMENTS}

This work was supported by the Biotechnology and Biological Sciences Research Council, [grant numbers BB/J014508/1 and BB/L013940/1] and H2020 MSCA ITN 2014: CLOSPORE, Grant No. 642068. We thank Alan Cockayne and Aleksandra Kubiak for their support and supervision, James Fothergill and Matthew Abbott for assistance with HPLC-MS analysis, Andrew Dempster for assistance with RNA extraction, David Ortega for assistance with C. pasteurianum, and all members of SBRC who helped to carry out this research.

\section{ABBREVIATIONS}

5' (UTR), 5'-untranslated region; CRISPR, clustered regularly interspaced short palindromic repeats; Cas, CRISPR-associated; sgRNA, single chimeric guide RNA; Pfdx, promoter of the ferredoxin gene; CAT, chloramphenicol acetyl transferase; TSS, transcriptional start site; SD, Shine-Dalgarno sequence; RBS, ribosome binding site; WT, wildtype; Pptb, promoter of the $C$. acetobutylicum ptb gene; ParaE, promoter of the C. acetobutylicum araE gene; RT-qPCR, reverse transcription quantitative polymerase chain reaction.

\section{REFERENCES}

(1) Minton, N. P., et al. (2016) A roadmap for gene system development in Clostridium. Anaerobe 41, 104-112.

(2) Carter, A. T., and Peck, M. W. (2015) Genomes, neurotoxins and biology of Clostridium botulinum Group I and Group II. Res. Microbiol. 166, 303-317.

(3) Uzal, F. A., et al. (2014) Towards an understanding of the role of Clostridium perfringens toxins in human and animal disease. Future Microbiol. 9, 361-377.

(4) Ofosu, A. (2016) Clostridium difficile infection: a review of current and emerging therapies. Ann. Gastroenterol. 29, 147-154.

(5) Sabra, W., Wang, W., Surandram, S., Groeger, C., and Zeng, A. (2016) Fermentation of mixed substrates by Clostridium pasteurianum and its physiological, metabolic and proteomic characterizations. Microb. Cell Fact., DOI: 10.1186/s12934-016-0497-4.

(6) Schwarz, K. M., et al. (2017) Towards improved butanol production through targeted genetic modification of Clostridium pasteurianum. Metab. Eng. 40, 124-137.

(7) Xue, C., Zhao, J., Chen, L., Yang, S.-T., and Bai, F. (2017) Recent advances and state-of-the-art strategies in strain and process engineering for biobutanol production by Clostridium acetobutylicum. Biotechnol. Adv. 35, 310-322.

(8) Hirano, K., et al. (2016) Enzymatic diversity of the Clostridium thermocellum cellulosome is crucial for the degradation of crystalline cellulose and plant biomass. Sci. Rep. 6, 35709.

(9) Maru, B. T., Munasinghe, P. C., Gilary, H., Jones, S. W., and Tracy, B. P. (2018) Fixation of $\mathrm{CO} 2$ and $\mathrm{CO}$ on a diverse range of carbohydrates using anaerobic, non-photosynthetic mixotrophy. FEMS Microbiol. Lett., DOI: 10.1093/femsle/fny039.

(10) $\mathrm{Xu}, \mathrm{H}$., et al. (2017) A study of $\mathrm{CO} /$ syngas bioconversion by Clostridium autoethanogenum with a flexible gas-cultivation system. Enzyme Microb. Technol. 101, 24-29.

(11) Kubiak, A. M., and Minton, N. P. (2015) The potential of clostridial spores as therapeutic delivery vehicles in tumour therapy. Res. Microbiol. 166, 244.

(12) Minton, N. P. (2003) Clostridia in cancer therapy. Nat. Rev. Microbiol. 1, 1-7.

(13) Bruder, M. R., Pyne, M. E., Moo-Young, M., Chung, D. A., and Chou, C. P. (2016) Extending CRISPR-Cas9 Technology from Genome Editing to Transcriptional Engineering in the Genus Clostridium. Appl. Environ. Microbiol. 82, 6109-6119.

(14) Pyne, M. E., Bruder, M. R., Moo-Young, M., Chung, D. A., and Chou, C. P. (2016) Harnessing heterologous and endogenous CRISPR-Cas machineries for efficient markerless genome editing in Clostridium. Sci. Rep. 6, 25666.

(15) Wasels, F., Jean-Marie, J., Collas, F., López-Contreras, A. M., and Lopes Ferreira, N. (2017) A two-plasmid inducible CRISPR/ Cas9 genome editing tool for Clostridium acetobutylicum. J. Microbiol. Methods 140, 5-11.

(16) Wang, Y., et al. (2015) Markerless chromosomal gene deletion in Clostridium beijerinckii using CRISPR/Cas9 system. J. Biotechnol. 200, $1-5$.

(17) Wang, S. (2018) Genome engineering of Clostridium difficile using the CRISPR-Cas9 system. Clin. Microbiol. Infect. 24, 1095.

(18) McAllister, K. N., Bouillaut, L., Kahn, J. N., Self, W. T., and Sorg, J. A. (2017) Using CRISPR-Cas9-mediated genome editing to generate $C$. difficile mutants defective in selenoproteins synthesis. Sci. Rep. 7, 14672.

(19) Shen, B., et al. (2014) Efficient genome modification by CRISPR-Cas9 nickase with minimal off-target effects. Nat. Methods $11,399-402$.

(20) $\mathrm{Xu}, \mathrm{T}$., et al. (2015) Efficient genome editing in Clostridium cellulolyticum via CRISPR-Cas9 nickase. Appl. Environ. Microbiol. 81, 4423-4431.

(21) Li, Q., et al. (2016) CRISPR-based genome editing and expression control systems in Clostridium acetobutylicum and Clostridium beijerinckii. Biotechnol. J. 11, 961-972.

(22) Groher, F., and Suess, B. (2014) Synthetic riboswitches - A tool comes of age. Biochim. Biophys. Acta, Gene Regul. Mech. 1839, 964973.

(23) Lynch, S. A., Desai, S. K., Sajja, H. K., and Gallivan, J. P. (2007) A high-throughput screen for synthetic riboswitches reveals mechanistic insights into their function. Chem. Biol. 14, 173-84.

(24) Cui, W., et al. (2016) Engineering an inducible gene expression system for Bacillus subtilis from a strong constitutive promoter and a theophylline-activated synthetic riboswitch. Microb. Cell Fact., DOI: 10.1186/s12934-016-0599-z.

(25) Rudolph, M. M., Vockenhuber, M. P., and Suess, B. (2015) Conditional control of gene expression by synthetic riboswitches in Streptomyces coelicolor. Methods Enzymol. 550, 283-299.

(26) Nakahira, Y., Ogawa, A., Asano, H., Oyama, T., and Tozawa, Y. (2013) Theophylline-dependent riboswitch as a novel genetic tool for strict regulation of protein expression in cyanobacterium Synechococcus elongatus PCC 7942. Plant Cell Physiol. 54, 1724-35.

(27) Dwidar, M., and Yokobayashi, Y. (2017) Controlling Bdellovibrio bacteriovorus gene expression and predation using synthetic riboswitches. ACS Synth. Biol. 6, 2035. 
(28) Topp, S., et al. (2010) Synthetic riboswitches that induce gene expression in diverse bacterial species. Appl. Environ. Microbiol. 76, 7881-4.

(29) Ohbayashi, R., Akai, H., Yoshikawa, H., Hess, W. R., and Watanabe, S. (2016) A tightly inducible riboswitch system in Synechocystis sp. PCC 6803. J. Gen. Appl. Microbiol. 62, 154-159.

(30) Heap, J. T., Pennington, O. J., Cartman, S. T., and Minton, N. P. (2009) A modular system for Clostridium shuttle plasmids. J. Microbiol. Methods 78, 79-85.

(31) Davis, J. H., Rubin, A. J., and Sauer, R. T. (2011) Design, construction and characterization of a set of insulated bacterial promoters. Nucleic Acids Res. 39, 1131-41.

(32) Bhattacharyya, S., et al. (2018) Accessibility of the ShineDalgarno Sequence Dictates N-Terminal Codon Bias in E. coli. Mol. Cell 70, 894-905.

(33) Mustoe, A. M., et al. (2018) Pervasive regulatory functions of mRNA structure revealed by high-resolution SHAPE probing. Cell 173, 181-195.

(34) Desai, S. K., and Gallivan, J. P. (2004) Genetic screens and selections for small molecules based on a synthetic riboswitch that activates protein translation. J. Am. Chem. Soc. 126, 13247-54.

(35) Huang, H., et al. (2016) CRISPR/Cas9-based efficient genome editing in Clostridium ljungdahlii, an autotrophic gas-fermenting bacterium. ACS Synth. Biol. 5, 1355-1361.

(36) Peters, J. M., et al. (2015) Bacterial CRISPR: accomplishments and prospects. Curr. Opin. Microbiol. 27, 121-6.

(37) Selle, K., and Barrangou, R. (2015) Harnessing CRISPR-Cas systems for bacterial genome editing. Trends Microbiol. 23, 225-232.

(38) Guo, C.-J. et al. (2018) Depletion of microbiome-derived molecules in the host using Clostridium genetics. bioRxiv DOI: $10.1101 / 401489$.

(39) Hong, W., Zhang, J., Cui, G., Wang, L., and Wang, Y. (2018) Multiplexed CRISPR-Cpf1-mediated genome editing in Clostridium difficile: toward the understanding of pathogenesis of C. difficile Infection. ACS Synth. Biol. 7, 1588-1600.

(40) Mougiakos, I., Bosma, E. F., Ganguly, J., van der Oost, J., and van Kranenburg, R. (2018) Hijacking CRISPR-Cas for highthroughput bacterial metabolic engineering: advances and prospects. Curr. Opin. Biotechnol. 50, 146-157.

(41) Sambrook, J., and Russell, D. W. (2001) Molecular Cloning: A Laboratory Manual, Cold Spring Harbor Laboratory Press.

(42) Shaw, W. V. (1975) Chloramphenicol acetyltransferase from chloramphenicol-resistant bacteria. Methods Enzymol. 43, 737-55.

(43) Silverstein, R. M. (1975) The determination of the molar extinction coefficient of reduced DTNB. Anal. Biochem. 63, 281-2. 